\title{
Design and simulation of a multienergy gamma ray absorptiometry system for multiphase flow metering with accurate void fraction and water-liquid ratio approximation
}

\begin{abstract}
Multiphase flow meters are used to measure the water-liquid ratio (WLR) and void fraction in a multiphase fluid stream pipeline. In the present study, a system of multiphase flow measurement has been designed by application of three thallium-doped sodium iodide scintillators and a radioactive source of ${ }^{133} \mathrm{Ba}$ simulated by Monte Carlo N-particle (MCNP) transport code. In order to capture radiations passing across the pipe, two direct detectors have been installed on opposite sides of the radioactive source. Another detector has been placed perpendicular to the transmission beam emitted from the ${ }^{133} \mathrm{Ba}$ source to receive radiations scattered from the fluid flow. Simulation was done by the MCNP code for different volumetric fractions of water, oil, and gas phases for two types of flow regimes, namely, homogeneous and annular; training and validation data have been provided for the artificial neural network (ANN) to develop a computation model for pattern recognition. Depending on applications of the neural system, several structures of ANNs are used in the current paper to model the flow measurement relations, while the detector outputs are considered as the input parameters of the neural networks. The first, second, and third structures benefit from two, three, and five multilayer perceptron neural networks, respectively. Increasing the number of ANNs makes the system more complicated and decreases the available data; however, it increases the accuracy of estimation of WLR and gas void fraction. According to the results, the maximum relative difference was observed in the scattering detector. It was clear that transmission detectors would demonstrate the difference between the flow regimes as well. It is necessary to note that the error calculated by the MCNP simulator is $<0.5 \%$ for the direct detectors (TR1 and TR2). Due to the difference between the data of the two flow regimes and the errors of data in the simulation codes of the MCNP, it was possible to separate these flow regimes. The effect of changing WLR on the efficiency for a constant void fraction confirms a considerable variance in the results of annular and homogeneous flows occurring in the scattering detector. There is a similar trend for the void fraction; hence, one can easily distinguish changes in efficiency due to the WLR. Analysis of the simulation results revealed that in the proposed structure of the multiphase flow meter and the computation model used for simulation, the two flow regimes are simply distinguishable.
\end{abstract}

Keywords: multiphase flow measurement $\bullet$ void fraction $\bullet$ water-liquid ratio $\bullet$ flow regime $\bullet$ MCNP $\bullet$ artificial neural network

\section{O. Khayat, H. Afarideh ${ }^{\bowtie}$}

Department of Energy Engineering and Physics

Amirkabir University of Technology

424 Hafez Ave., 15875-4413, Tehran, Iran

E-mail: Hafarideh@aut.ac.ir

Received: 6 June 2018

Accepted: 14 December 2018

\section{Introduction}

A multiphase flow meter is a single standing device or a combination of various tools, which quantifies the volumetric flow rates of water, oil, and gas phases separately in a multiphase fluid stream. In a multiphase fluid pipeline, the pattern of flow is unknown; practically, we must deal with an indistinctive pattern of a three-phase flow with varying volumetric fractions of the phases $[1,2]$. Measuring the multiphase flow by means of phase separation is not always feasible [3]. Gamma densitometry is a helpful method in this context; however, as the fluid flow regime becomes intermittent or annular, the measurement accuracy falls considerably. Tra- 
ditional gamma densitometry includes inserting a detector opposite a high-energy source (cesium) to measure the volumetric fractions. Furthermore, it is necessary to use double- or multiple-energy sources, which requires sensitive detectors. Another method described involves installing multiple detectors opposite the source so that volumetric fractions of the phases and the flow regime are evaluated by measuring the attenuation rates at each individual detector. This is done by placing a collimator opposite the source and the detectors so that they only capture the attenuated energies, not the dispersed ones. The main phenomena occurring during gamma attenuation are the photoelectric and Compton effects. The Compton effect is a function of the fluid density, and the photoelectric phenomenon, which is dominant at lower energy levels, strongly depends on the atomic number of the material, so that gamma ray attenuation is exponentially proportional to four or five times the atomic number of the material; hence, as water salinity increases, chlorine - with its high atomic number - intensifies the attenuation rate and leads to significant errors in estimations.

In two-phase flux measurement systems, the pipeline wall and the flowing fluid would absorb gamma rays. The source and the detectors are placed on opposite sides of the cross section of the pipe. The collimator rectifies the beams alongside the diameter of the pipe. Figure 1 illustrates the layout of the source and the detector [4].

The problems with the size, as well as the installation and maintenance costs, of conventional multiphase flow meters - which measure each single-phase flow after separation of the phases by multiphase separators - increase the attention given to new methods of flow metering. In this regard, the flow-metering method called gamma ray densitometry, which is based on gamma ray attenuation, has become very popular recently. Gamma ray densitometry has been used successfully in multiphase flow measurement and well-monitoring applications in the oil and gas industry $[5,6]$. The degree of gamma ray attenuation in multiphase flow metering depends on the density and the adsorption coefficient as the inherent characteristics of the material through which the gamma ray passes, the distance that the gamma ray passes, and the gamma ray wavelength.

Precise multiphase flow measurement using gamma ray densitometry depends on accurate flow regime identification. Identification of flow regimes in operational conditions is a difficult task in the

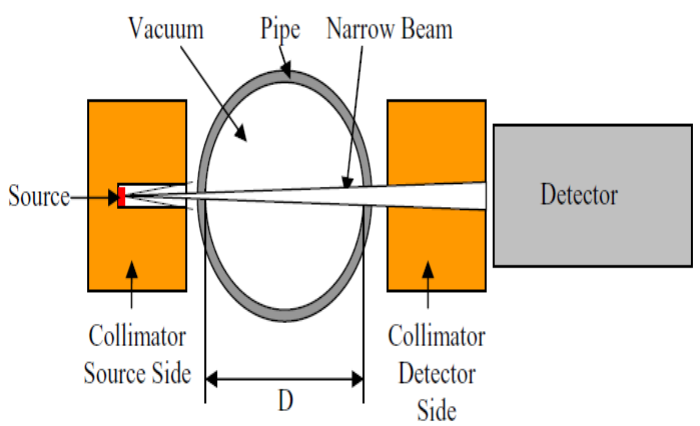

Fig. 1. Schematics of the source and the detector. flow measurement process. On the other hand, identifying the flow regimes has a great importance in multiphase transportation, especially in offshore petroleum exploitation. Thus, finding a system that can reduce the complication of flow regime identification and provide precise prediction of the volume fraction of each phase has been one of the major concerns of researchers in recent years [7].

Nazemi et al. [8] applied the radial basis function (RBF), the artificial neural network (ANN), and the broad beam gamma ray attenuation technique for the identification of the flow regime and measurement of the void fraction (VF) under two-phase flow condition. They used Monte Carlo simulation to provide training and test data for the neural network. The results of their study indicate that ANN can be used for precise prediction of the volume fraction and identification of the flow regime in a two-phase flow.

Collimators are used basically to prevent the absorption of unfavourable radiations at the detectors. Nevertheless, complete prevention is not practicable [2]. Johansen and Jackson used a detector $D t$ and a receiver $D s$ in their assembly to measure and compare the absorbed energy for their volumetric analysis. Formerly, the gas void fraction (GVF) was evaluated by placing a detector opposite a high-energy source such as Cs and recording the absorbed energy [9].

$$
\mathrm{GVF}=\frac{\ln \left(I_{\text {mix }} / I_{\text {liquid }}\right)}{\ln \left(I_{\text {gas }} / I_{\text {liquid }}\right)}
$$

where $I_{\text {mix }}$ is the energy intensity of the multiphase fluid, $I_{\text {gas }}$ and $I_{\text {liquid }}$ are the energy intensities when the pipe's cross-sectional area is full of gas and water, respectively. Later, they combined two detectors in a model called dual densitometer (DMD) (Fig. 2) [9].

Detector $D s$ is set somewhere between the source and $D t$. In order to see the Compton effect at detector $D s$, a source in which both the Compton effect and the photoelectric phenomenon are dominant must be used; hence, it is not practical to use Cs as the source because its energy is very high and the effect of the photoelectric phenomenon is very weak. Therefore, americium of 122 millicurie (mCi) radiation level was used as the source, in which both the Compton effect and the photoelectric phenomenon are dominant. To measure the intensity at the detectors, they used an apparatus containing multiple receivers at various angles (Fig. 3).

A collimator was used for the detector $D t$ to reduce the buildup factor. For the detector $D s$, two methods could be applied: either ignoring

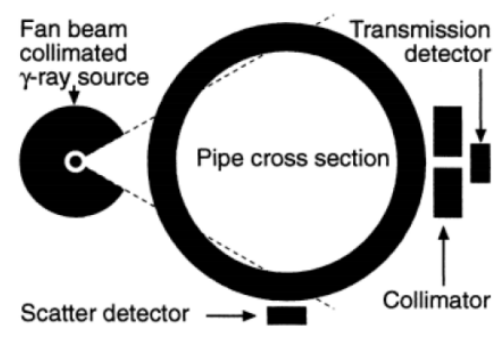

Fig. 2. DMD with two detectors. 


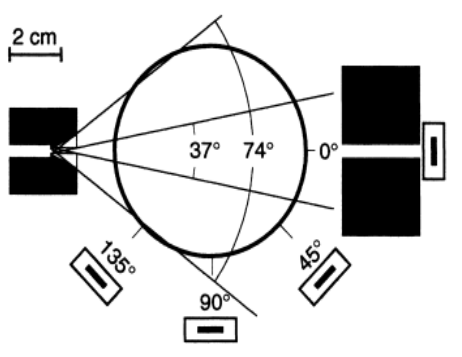

Fig. 3. DMD densitometry: multiple receivers at various angles.

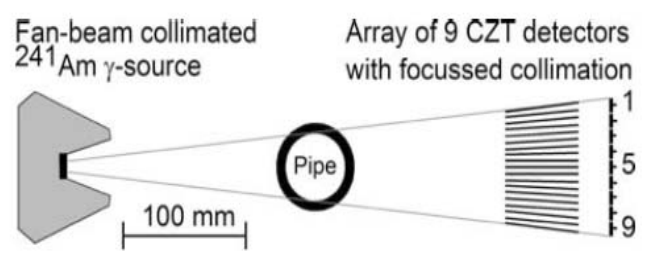

Fig. 4. Schematics of the measurement system at the University of Bergen.

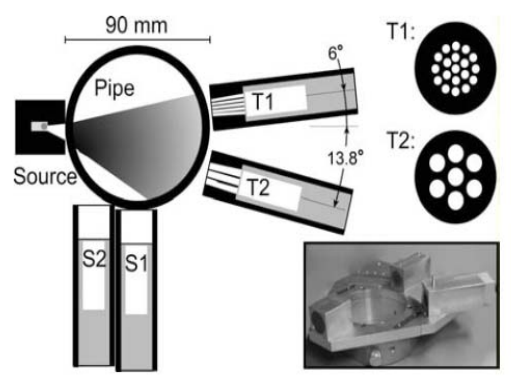

Fig. 5. Two-detector array of the University of Bergen.

the collimator to optimize the numeration, or using collimators to reduce the scattering effects. In order to conduct the experiment, the source was irradiated under $15^{\circ}$ and $54^{\circ}$, called narrow and wide radiations, respectively [9]. CdZnTe (CZT) detectors with dimensions of $12 \times 12 \times 12 \mathrm{~mm}$ are strong deterrents to measure energy intensity. The $D s$ detectors were held at $45^{\circ}, 90^{\circ}$, and $135^{\circ}$ angles. Two flow regimes were investigated, namely, homogeneous and annular flows. As was expected, at $135^{\circ}$ angle, the detectors $D t$ and $D s$ showed the maximum and minimum sensitivities, respectively. Using a wide radiation range increased the measurement area at the pipe and provided helpful information about the flow regime. The authors defined a correlation to calculate the ratio of energy intensity at detector $D t$ to that at detector $D s$; it becomes a constant ratio, $R$.

$$
R=\frac{I_{s}}{I_{T}^{n}}
$$

where $R$ is a function of the gas volumetric fraction and is independent of water salinity. The value of $n$ is approximately 0.55 ; however, it depends on the geometrical configuration [9]. In a research in 1993, a single-energy ${ }^{137} \mathrm{Cs}$ source and a sodium iodide detector were used in a $9.525 \mathrm{~mm}$ two-phase (liquid-gas) pipeline to determine the flow regimes. For multiphase flow analysis, a Ge-Li detector and multiple-energy gamma ray sources of cobalt-57 $(122 \mathrm{keV})$ and barium-133 (365 keV) were set on a pipeline of $0.1 \mathrm{~m}$ diameter [2]. Later, in another research project, two sources of ${ }^{137} \mathrm{Cs}$ (662 keV) and americium-241 (59.5 keV), as well as a sodium iodide detector, were used [9]. A research project was conducted at the University of Bergen (Norway), in which the arrangements shown in Figs. 4 and 5 were used to evaluate the phase fractions [10]. A CZT semiconductor detector was used in that research. The area of each of the detectors of this array was $1 \mathrm{~cm}^{2}$. A CsI (Na) two-phase detector, with dimensions of $13 \times 38 \mathrm{~mm}$, was used for flow regime determination. Americium-241 was set as the source (Fig. 4).

The geometry of the system comprises the following (Fig. 6):

- a radioactive barium-133 source of $10 \mathrm{mCi}$ radioactivity;

- three 1-inch $\mathrm{NaI}(\mathrm{Tl})$ horizontally installed scintillation detectors on the pipe's cross-sectional plane;

- a 2 inch vertically aligned pipeline carrying water, oil, and gas.

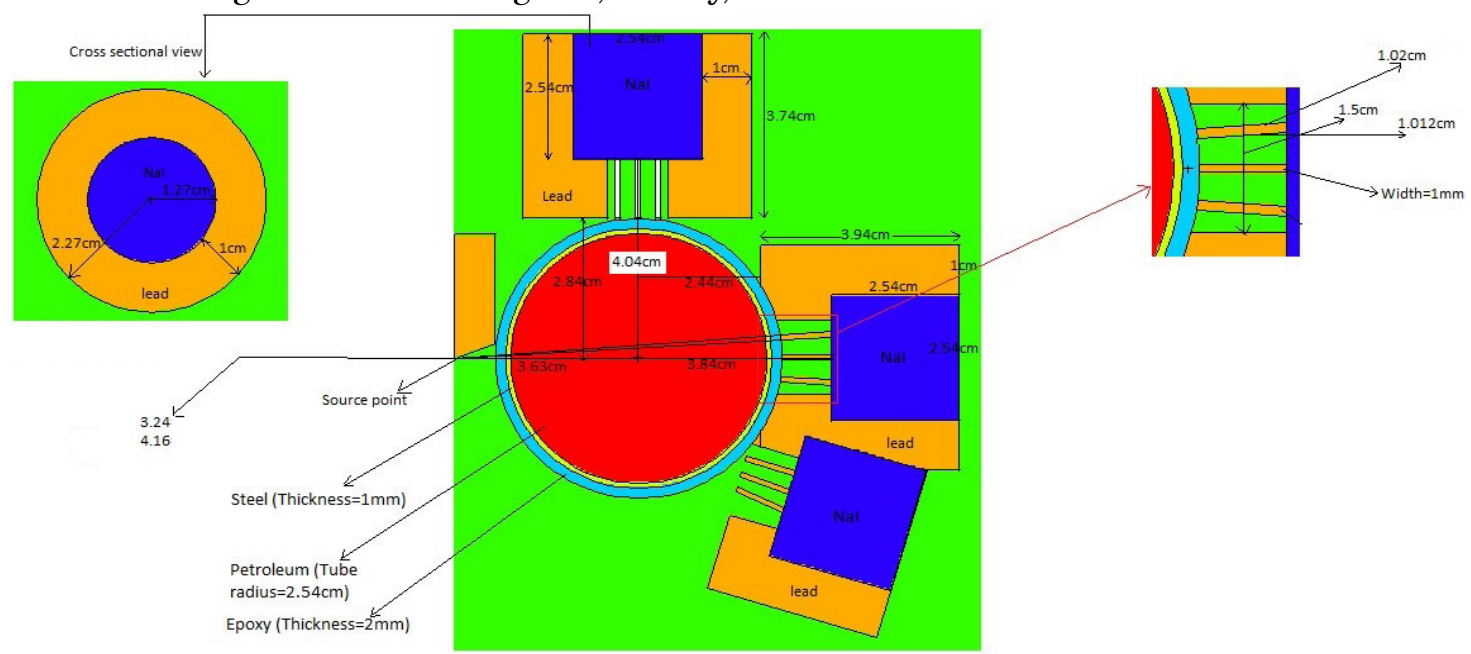

Fig. 6. The general geometry of the measurement system: hydrocarbon content inside tube (red section), tube steel wall (green narrow ring surrounding hydrocarbon section), epoxy wall (blue ring surrounding steel wall), NaI cylindrical scintillation detectors (blue sections), as well as lead shields and collimators (orange sections). 


\section{Materials and methods}

\section{Conventional flow patterns}

In the present project, two conventional flow patterns, namely, homogeneous and annular flows, were simulated. Densities of water, oil, and gas were assumed to be $1.1,0.85$, and $0.05 \mathrm{~g} / \mathrm{cm}^{3}$. The gas phase was $\mathrm{CH}_{4}$, and water was pure $\mathrm{H}_{2} \mathrm{O}$. The constitutive elements of the oil phase were hydrogen, carbon, oxygen, nitrogen, and sulphur.

- Homogeneous pattern: the three phases (water, oil, and gas) were mixed homogeneously.

- Annular pattern: the gas was assumed to be the carrier phase passing axially through the middle of the pipeline. Oil and water formed a homogeneous thin film around the perimeter of the pipe, surrounding the gas phase.

\section{Estimation of flow type and the WLR fraction using ANNs}

Artificial neural networks (ANNs) constitute a conventional technique for the classification and analysis of data. Processing various data in the ANNs may yield helpful information about the system; hence, data adjustment plays a significant role in their application [11-14]. An ANN is defined as an input-output system in which hidden layers do the required processes. Therefore, all of the output systems with an ANN structure are composed of the following constituents:

- input layer: including independent variables vector;

- hidden layers: including operator functions, input data processing neurons;

- output layer: including estimation of the target variable vector.

Figure 7 illustrates a simple ANN with a single hidden layer.

Training refers to evaluation of the coefficients or setting their weights in the networks. The goal is to minimize the sum of the squared errors, i.e., minimizing the difference between the real data and the calculated output of the ANNs.

Data points are classified into three categories. The first class of data is used for training the network. The second class is a measure of the validation. Provided that the ANN verifies the validation steps, the third class of data is used to assess the performance of the network and calculate its error [15].

For flow metering purposes, multilayer perceptron neural networks are being mostly used. This

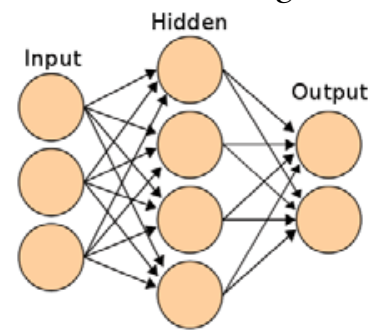

Fig. 7. Simple ANNs with a single hidden layer. type of networks is composed of neurons in multiple layers usually correlated with a feed-forward algorithm. Often a sigmoid function is used as the transfer function. It has a continuous derivative, allows for a backpropagation algorithm, and is simply differentiable.

$$
\begin{aligned}
& f(x)=\frac{1}{1+e^{-x}} \\
& y^{\prime}=y(1-y)
\end{aligned}
$$

There are various well-known methods for training the ANNs. The backpropagation algorithm is a popular practical training technique to minimize errors [16].

In the current paper, the multilayer perceptron and Levenberg-Marquardt (1978) training algorithms were used [17]. Depending on the number of ANNs and their corresponding structures, different operations were followed. The following would represent the three main structures and their simulation results (Fig. 8). All of the three structures use a single independent ANN to recognize the flow regime [14]. Results confirmed that this ANN was capable of making estimations with $100 \%$ accuracy.

\section{Different structures of the ANNs}

The first structure consisted of an ANN for flow regime identification and another network for estimation of the GVF and the water-liquid ratio (WLR).

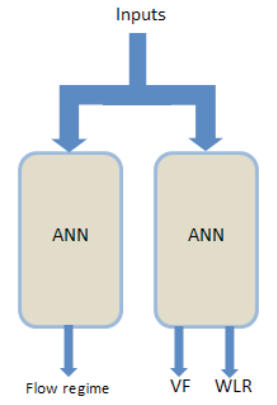

(a)

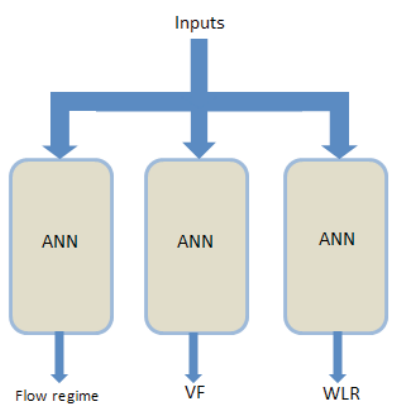

(b)

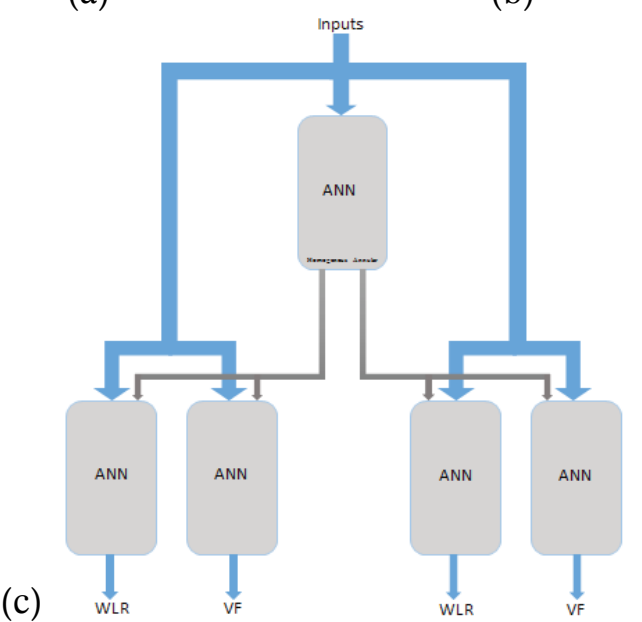

Fig. 8. Schematic representation of (a) first structure, (b) second structure, and (c) third structure of the ANNs for flow regime identification and VF and WLR estimation. 
The second structure was made up of an ANN for determination of the flow regime, a network for estimation of the GVF, and another one for evaluation of WLR.

The third structure consisted of five ANNs. One for flow regime recognition; then, for each of the flow regimes, there were two ANNs for evaluation of the VF and WLR, respectively.

There were 209 data points generated by the Monte Carlo N-particle (MCNP) transport simulator software. In the first structure, 147 data points (70\%) were used for training the ANNs and $31(15 \%)$ for validation purpose to avoid overfitting. Further, 31 data points $(15 \%)$ were used for evaluation of the network performance and calculation of errors. For the other structures, 167 data points $(80 \%)$ were taken for training and $21(10 \%)$ for validation to avoid overfitting problems. Finally, 21 data points $(10 \%)$ were used to analyse the results and find the estimation errors. It is important to note that patterns containing only one material (e.g., 100\% air) were removed from the training set because they represent the same flow regime and might expose the ANNs to deviation. Inputs to the ANNs were the following:

1. The number of counts at the first detector (TR1) for four different ranges of energy.

2. The number of counts at the second detector (TR2) for four different ranges of energy.

3. The total number of counts at the scattering detector (total SCA).

Outputs of the ANNs were as follows:

1. For the ANN that recognizes the flow regime, the output could be either zero or one.

2. For ANNs that evaluate the VF and WLR, the outputs are equal to the estimates of the target value for the corresponding input data.

Overall, nine inputs are given to each of the networks. The outputs of the ANNs for flow regime identification are simply a single digit: either ' 1 ' if the flow is annular, or ' 0 ' if the flow is homogeneous.

Mean squared error (MSE) and/or root mean squared error (RMSE) was used to evaluate the simulation accuracy of the networks.

$$
\text { MSE }=\sum_{t=1}^{n} \frac{\left(y_{t}-\hat{y}_{t}\right)^{2}}{n}
$$

$$
\mathrm{RMSE}=\sqrt{\sum_{t=1}^{n} \frac{\left(y_{t}-\hat{y}_{t}\right)^{2}}{n}}
$$

As was mentioned formerly, one could observe that ANNs having those nine inputs were able to recognize the flow regime with $100 \%$ accuracy (Fig. 9). The structures and their results are described in the "Results and discussion" section.

\section{Results and discussion}

\section{Effects of flow regime on results}

The variances in the results for homogeneous and annular flows with 10\% VF and 0\% WLR are given in Table 1.

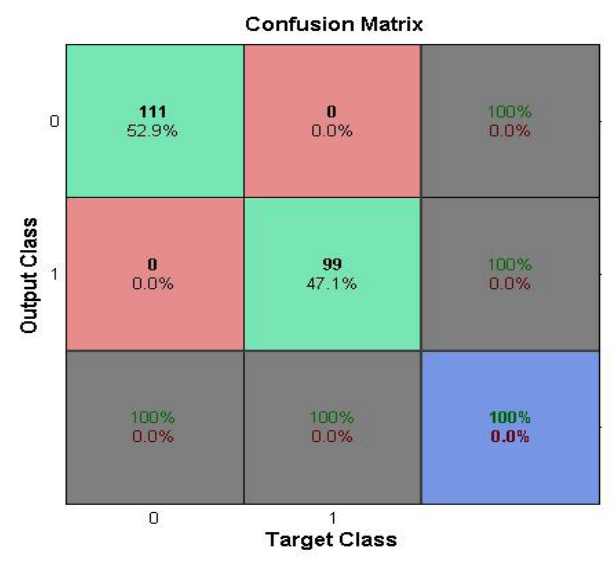

Fig. 9. The confusion matrix for flow regime recognition.

Table 1. Variance in the results

\begin{tabular}{cccc}
\hline \multicolumn{4}{c}{ \% Variance in different regimes $(\mathrm{VF}=10 \%, \mathrm{WLR}=0 \%)$} \\
\hline Energy $(\mathrm{keV})$ & TR1 & TR2 & SCA \\
\hline 81 & 17.96632 & 3.729679 & - \\
276 & 12.45799 & 2.988704 & - \\
303 & 11.80512 & 2.783990 & - \\
356 & 11.36379 & 2.321706 & - \\
384 & 10.52108 & 1.992711 & - \\
$20-60$ & 9.413337 & 4.399542 & - \\
$60-100$ & 14.52055 & 4.014705 & - \\
$100-300$ & 8.135009 & 4.181744 & - \\
Above 300 & 10.70055 & 2.209990 & - \\
Total & 10.02833 & 3.550210 & 9.234494 \\
\hline
\end{tabular}

According to Table 1, the maximum relative difference was observed in the scattering detector. It was clear that other detectors would demonstrate the difference between the flow regimes as well. It is necessary to note that the error calculated by the MCNP simulator is $<0.5 \%$ for the direct detectors (TR1 and TR2). Due to the difference between the data of the two flow regimes and the errors of the data in the simulation codes of the MCNP, it was possible to separate these flow regimes.

The effect of change in the WLR on the efficiency for a constant VF of 10\% is shown in Fig. 10. The numbers confirm a considerable variance in the results of annular and homogeneous flows occurring in the scattering detector. There is a similar trend for

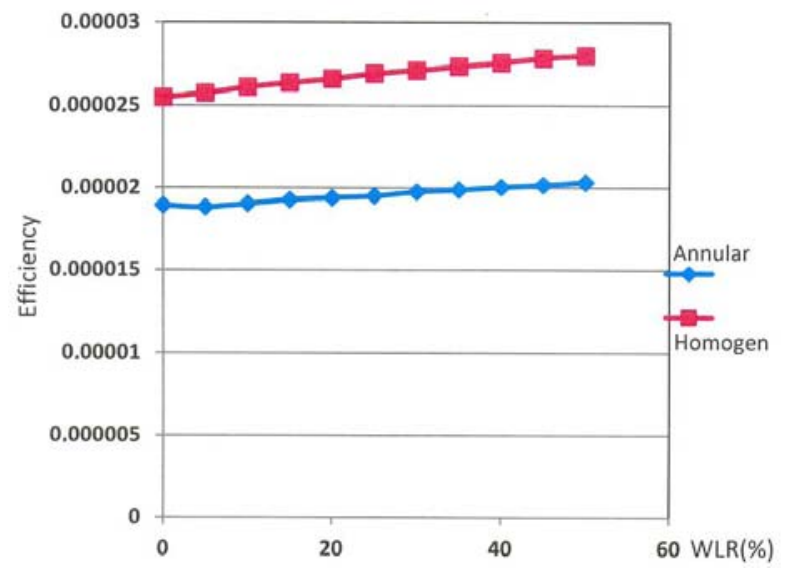

Fig. 10. Effect of changing flow regimes on efficiency due to variations of $\mathrm{WLR}$ for $\mathrm{VF}=10 \%$. 
(a)
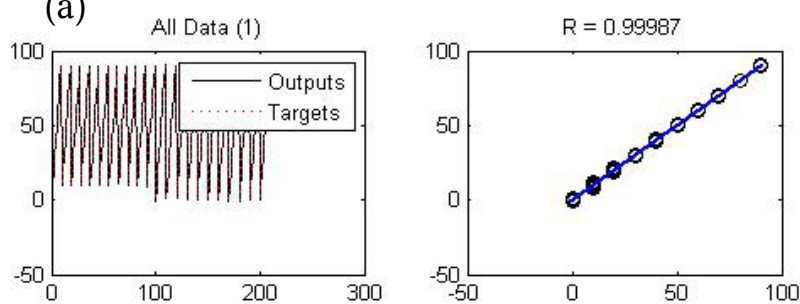

MSE $=0.19894$, RMSE $=0.44603$

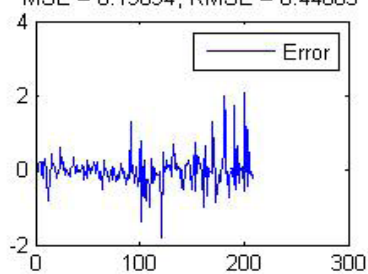

(b)

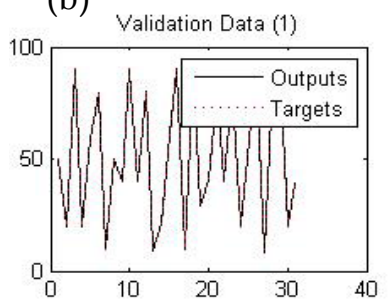

MSE $=0.37059$, RMSE $=0.60876$

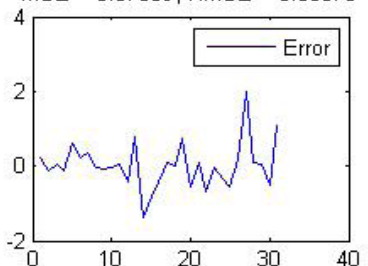

(c)

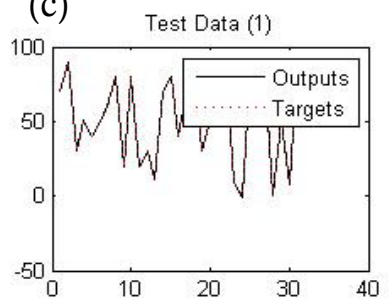

MSE $=0.29454$, RMSE $=0.54271$

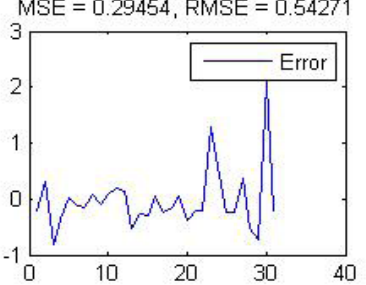

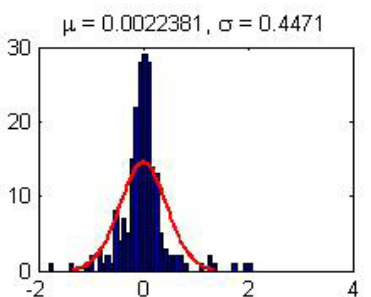
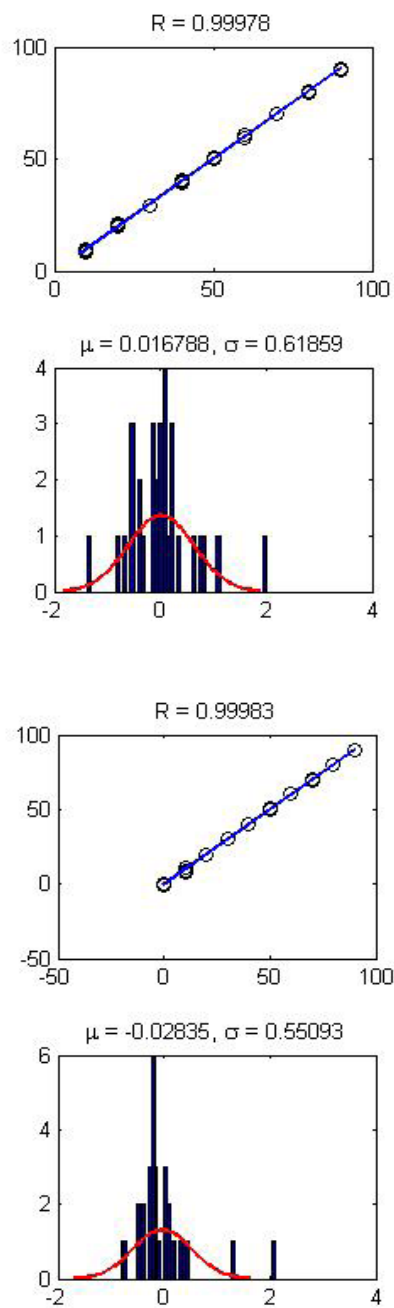

Fig. 11. First structure: (a) total data for estimation of VF; (b) validation data for VF estimation; (c) test data for VF estimation.

other VFs; hence, one can easily distinguish changes in efficiency due to the WLR.

Analysis of the simulation results revealed that the two flow regimes are simply detectable. Furtheramore, there is a considerable variance in the results, which simplifies flow regime recognition.

\section{First structure}

For ANNs with the first structure, there were two outputs. One of them showed the VF and another one showed the WLR, indicating that two neurons were established at the output layer and nine at the input layer.

Simulation results of the first structure are given in Figs. 11 and 12.

Based on the figures, RMSE on the test data set for VF estimation is about 0.54 . The correlation between the outputs of the ANNs and the targets is very close.

Diagrams of the WLR measurements are presented in Fig. 12.

As shown in the diagrams, the RMSE value for the test data used in the estimation of the WLR is about 2.8. The outputs of the ANN and the target values are compared in Table 2.

\section{Second structure}

In the second structure, three ANNs were considered for making estimations. A network was set in charge of the flow regime determination; the other two networks with nine input neurons and one output neuron were organized to calculate the volumetric fractions and WLR values. (a)
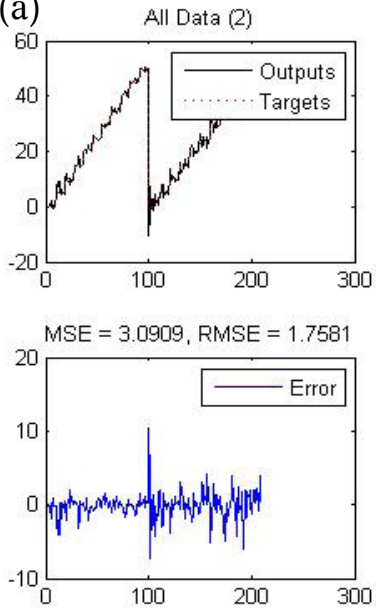

(b)
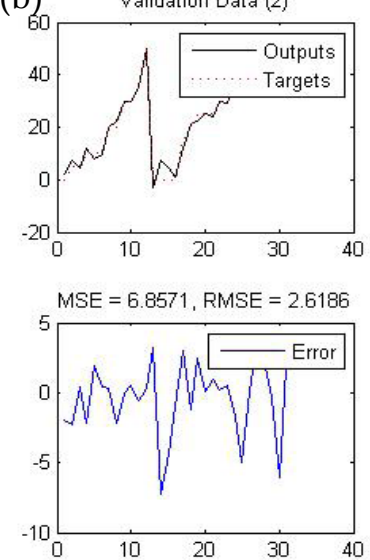
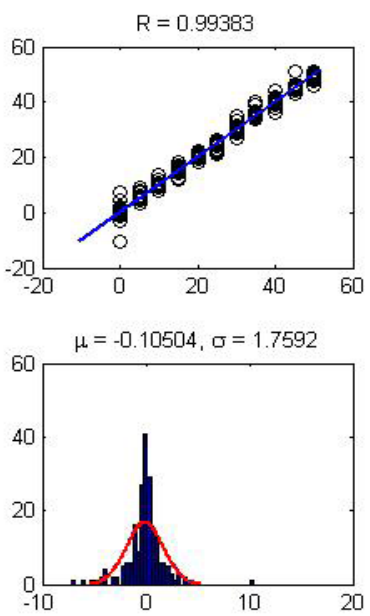

$\mathrm{R}=0.98609$

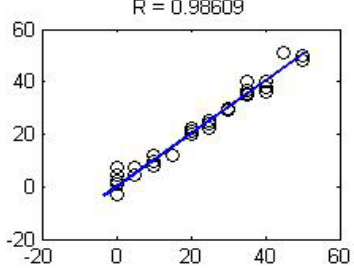

$\mu=-0.42673, \sigma=2.6263$

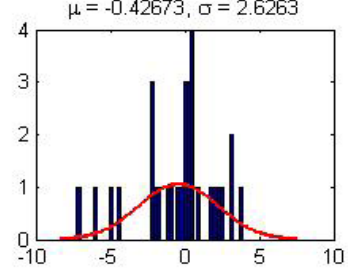

Fig. 12. First structure: (a) total data for estimation of WLR; (b) validation data for estimation of WLR. 
Table 2. Comparison of test outputs (ANN results) with test targets (MCNP results) during VF and WLR estimation in the first structure of ANNs

\begin{tabular}{|c|c|c|c|}
\hline \multicolumn{2}{|c|}{ VF } & \multicolumn{2}{|c|}{ WLR } \\
\hline $\begin{array}{c}\text { Test targets } \\
\text { (1) }\end{array}$ & $\begin{array}{c}\text { Test outputs } \\
\text { (1) }\end{array}$ & $\begin{array}{c}\text { Test targets } \\
\text { (2) }\end{array}$ & $\begin{array}{c}\text { Test outputs } \\
\text { (2) }\end{array}$ \\
\hline$\overline{70}$ & 70.22 & 0 & -0.86 \\
\hline 90 & 89.70 & 0 & 0.07 \\
\hline 30 & 30.81 & 5 & 9.01 \\
\hline 50 & 50.36 & 5 & 8.97 \\
\hline 40 & 39.98 & 10 & 9.28 \\
\hline 50 & 50.13 & 15 & 18.19 \\
\hline 60 & 60.16 & 15 & 16.60 \\
\hline 80 & 79.93 & 15 & 15.00 \\
\hline 20 & 20.08 & 20 & 20.78 \\
\hline 80 & 79.92 & 20 & 20.20 \\
\hline 20 & 19.80 & 30 & 29.10 \\
\hline 30 & 29.87 & 30 & 28.49 \\
\hline 10 & 10.52 & 35 & 36.11 \\
\hline 70 & 70.27 & 35 & 35.49 \\
\hline 80 & 80.29 & 35 & 35.40 \\
\hline 40 & 39.94 & 40 & 40.45 \\
\hline 70 & 70.23 & 40 & 41.23 \\
\hline 80 & 80.16 & 40 & 39.29 \\
\hline 30 & 29.95 & 45 & 44.90 \\
\hline 50 & 50.38 & 45 & 45.95 \\
\hline 60 & 60.20 & 45 & 46.42 \\
\hline 90 & 90.22 & 45 & 45.15 \\
\hline 10 & 8.71 & 50 & 48.48 \\
\hline 0 & -0.44 & 0 & -10.37 \\
\hline 70 & 70.24 & 0 & 0.24 \\
\hline 80 & 80.23 & 0 & 2.09 \\
\hline 70 & 69.63 & 25 & 20.97 \\
\hline 0 & 0.52 & 30 & 35.20 \\
\hline 50 & 50.73 & 40 & 44.35 \\
\hline 10 & 7.91 & 50 & 51.35 \\
\hline 80 & 80.21 & 50 & 45.99 \\
\hline
\end{tabular}

Results of the simulation for VF calculation are shown in Fig. 13 and Table 3.

According to the diagrams of the test data, the RMSE is equal to 0.29 , and in the worst case, the error value approaches 1\%. Meanwhile, the correlation between the inputs and the outputs is very close to unity. In a histogram of accuracy, the error is negligible; in fact, it approaches zero. Results confirmed the generalized and efficient performance of the ANNs.

Simulation results of the estimation of WLR are illustrated in Fig. 14 and Table 3.

Based on the diagrams, the RMSE for the test data in the estimation of WLR is 1.8 , and the error is $<5 \%$ in the worst scenario. There is a very strong correlation between input and output data; meanwhile, in the histogram, the error is very close to zero. It was expected that the estimation error in the evaluation of WLR will be higher than that for VFs because the densities of water and oil are much closer compared with a water-air two-phase system; hence, the capability for generalization of the networks was diminished.

Figures 13 and 14 show the outputs of ANNs and the target values. (a)
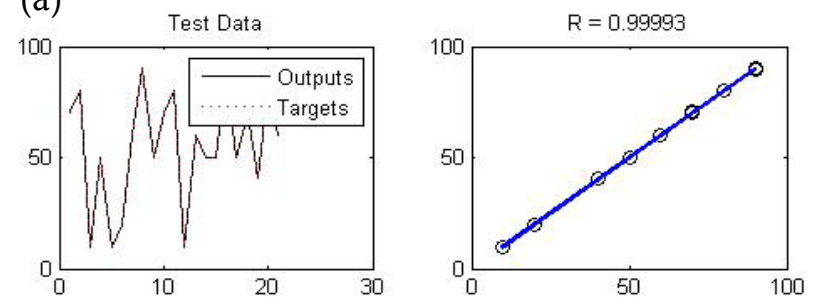

MSE $=0.089802$, RMSE $=0.29967$
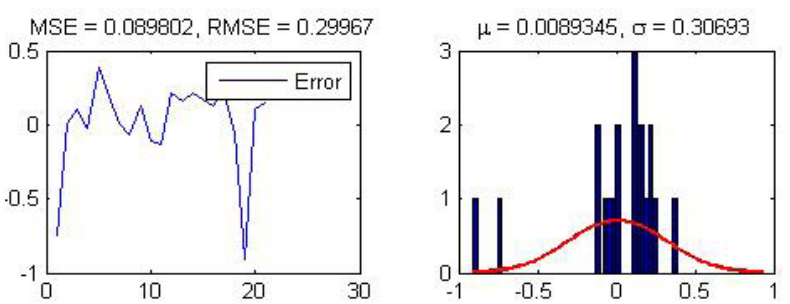

(b)
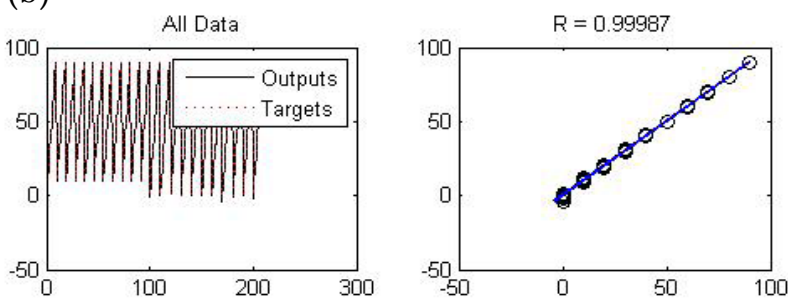

MSE $=0.20303$, RMSE $=0.45059$
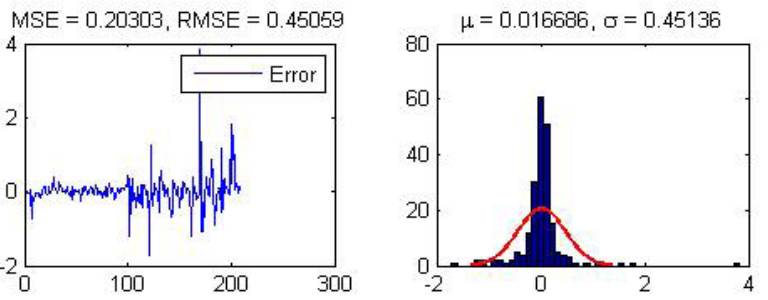

(c)
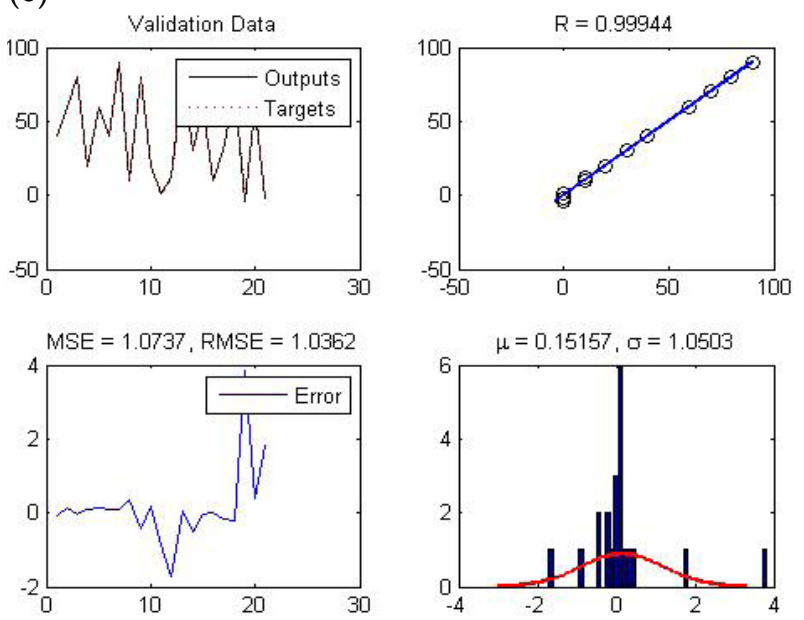

Fig. 13. Second structure: (a) total data for estimation of VF; (b) validation data for estimation of the VF; (c) test data for estimation of the VF.

\section{Third structure}

As was formerly described, five ANNs are used in the third structure: one ANN is used to recognize the flow regime, and the others are used to evalu- 
(a)

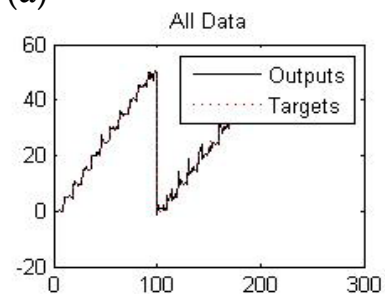

MSE $=1.3586$, RMSE $=1.1656$

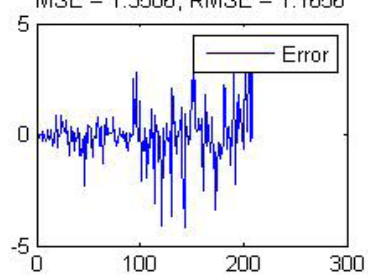

(b)

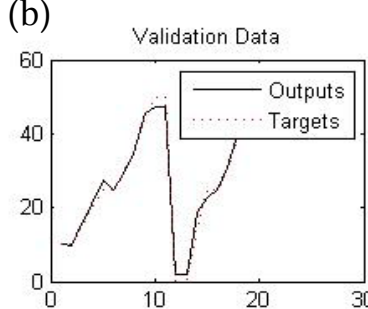

MSE $=3.3103$, RMSE $=1.8194$

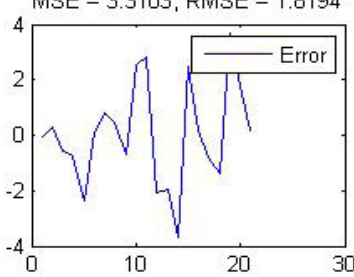

(c)

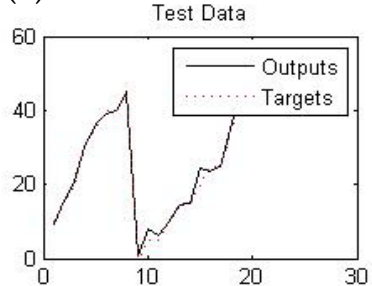

MSE $=3.4975$, RMSE $=1.8702$

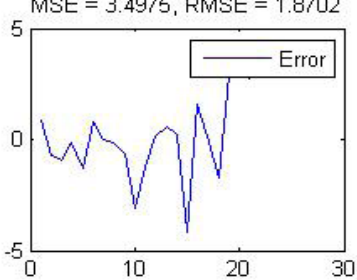

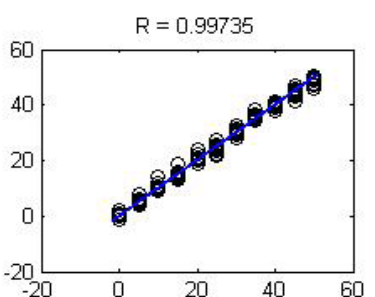
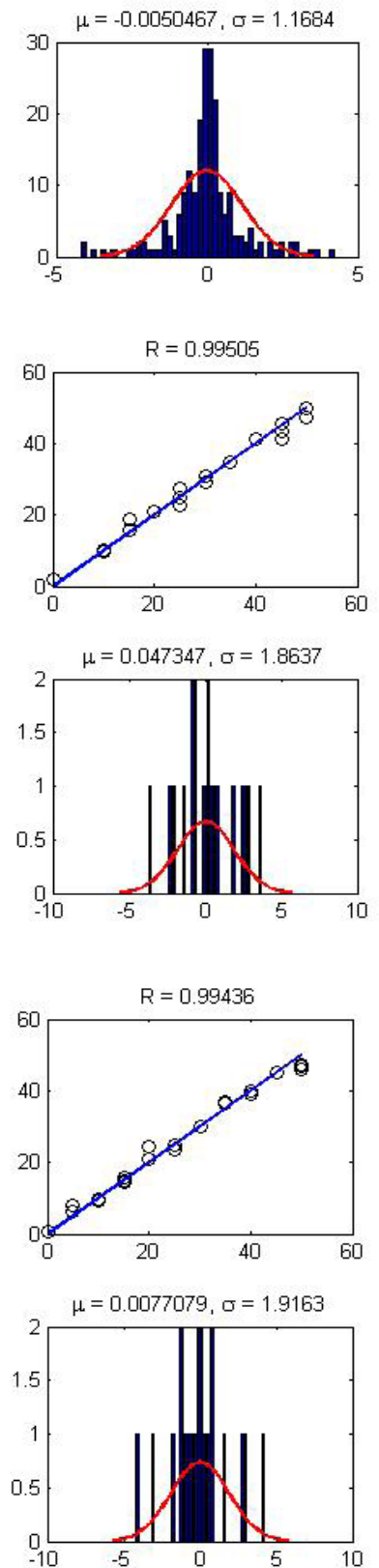

Fig. 14. Second structure: (a) total data for estimation of WLR; (b) validation data for estimation of WLR; (c) test data for estimation of WLR.

ate the VF and WLR for either of the flow patterns (homogeneous or annular).

For the homogeneous flow regime, there were totally 110 data points: $10 \%$ (11 data points) were used for validation, $10 \%$ for test, and $80 \%$ (88 data points) for training.

Results of the simulation for estimation of the $\mathrm{VF}$ in homogeneous flow conditions are shown in Fig. 15.
Table 3. Comparison of test outputs (ANN results) and test targets (MCNP results) for VF and WLR estimation in the second structure of ANNs

\begin{tabular}{lrcc}
\hline \multicolumn{2}{c}{ VF } & \multicolumn{2}{c}{ WLR } \\
\hline Test targets & Test outputs & Test targets & Test outputs \\
\hline 70 & 70.75 & 10 & 9.17 \\
80 & 79.99 & 15 & 15.75 \\
10 & 9.89 & 20 & 20.96 \\
50 & 50.02 & 30 & 30.18 \\
10 & 9.61 & 35 & 36.27 \\
20 & 19.81 & 40 & 39.18 \\
60 & 59.99 & 40 & 40.01 \\
90 & 90.07 & 45 & 45.15 \\
50 & 49.87 & 0 & 0.61 \\
70 & 70.11 & 5 & 8.10 \\
80 & 80.13 & 5 & 6.20 \\
10 & 9.79 & 10 & 9.86 \\
60 & 59.84 & 15 & 14.39 \\
50 & 49.78 & 15 & 14.78 \\
50 & 49.83 & 20 & 24.19 \\
90 & 89.87 & 25 & 23.42 \\
50 & 49.77 & 25 & 24.89 \\
70 & 70.05 & 35 & 36.70 \\
40 & 40.91 & 50 & 47.03 \\
80 & 79.89 & 50 & 45.78 \\
60 & 59.85 & 50 & 47.25 \\
\hline
\end{tabular}

Table 4. Comparison of test outputs (ANN results) and test targets (MCNP results) for estimation of the VF and the WLR for a homogeneous flow regime in the third structure of ANNs

\begin{tabular}{lccc}
\hline \multicolumn{2}{c}{ VF } & \multicolumn{2}{c}{ WLR } \\
\hline Test targets & Test outputs & Test targets & Test outputs \\
\hline 70 & 70.06 & 0 & -0.05 \\
50 & 50.03 & 0 & -0.62 \\
60 & 59.89 & 5 & 10.00 \\
60 & 59.88 & 20 & 19.58 \\
90 & 90.22 & 20 & 20.90 \\
0 & -0.69 & 20 & 19.38 \\
20 & 21.18 & 25 & 24.05 \\
10 & 9.38 & 30 & 29.75 \\
30 & 29.31 & 45 & 46.10 \\
50 & 49.38 & 50 & 49.15 \\
90 & 89.92 & 50 & 49.33 \\
\hline
\end{tabular}

Table 5. Comparison of test outputs (ANN results) and test targets (MCNP results) for estimation of the VF and the WLR for annular flow regime in the third structure of ANNs

\begin{tabular}{lccc}
\hline \multicolumn{2}{c}{ VF } & \multicolumn{2}{c}{ WLR } \\
\hline Test targets & Test outputs & Test targets & Test outputs \\
\hline 60 & 60.19 & 5 & 3.68 \\
90 & 90.06 & 10 & 9.72 \\
50 & 50.08 & 25 & 25.70 \\
30 & 30.03 & 35 & 34.67 \\
80 & 80.00 & 35 & 33.42 \\
20 & 20.10 & 35 & 35.52 \\
50 & 50.02 & 40 & 39.77 \\
60 & 60.08 & 40 & 40.07 \\
10 & 9.97 & 50 & 49.93 \\
40 & 39.95 & 50 & 46.97 \\
\hline
\end{tabular}


(a)
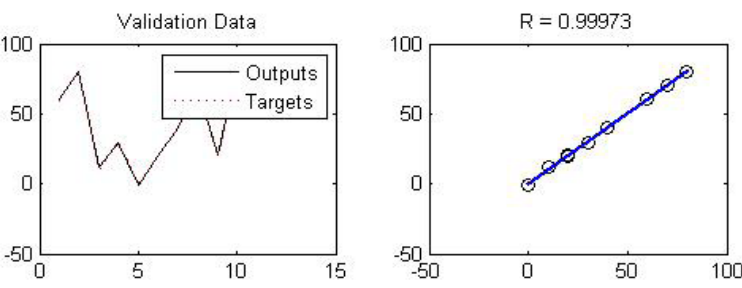

MSE $=0.49134$, RMSE $=0.70096$

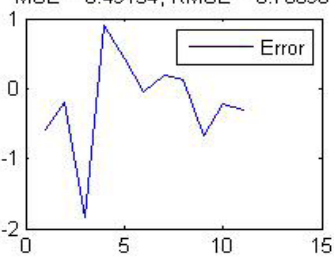

(c)

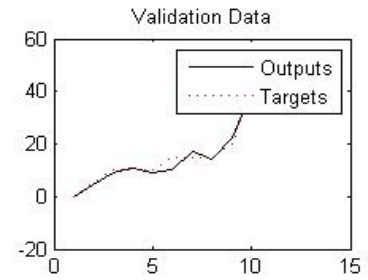

MSE $=3.9159$, RMSE $=1.9789$

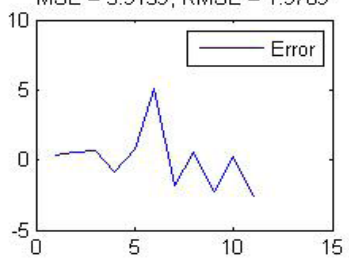

$\mu=-0.20326, \sigma=0.70358$

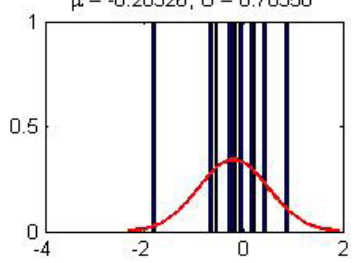

$R=0.99239$
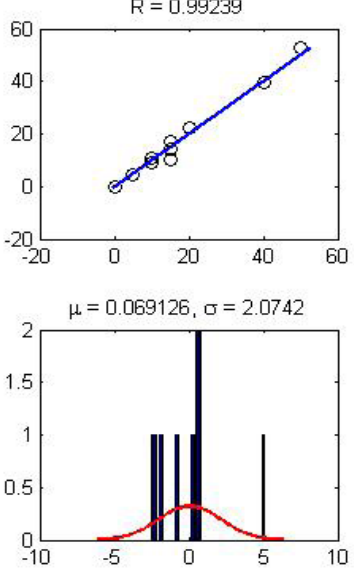

(b)
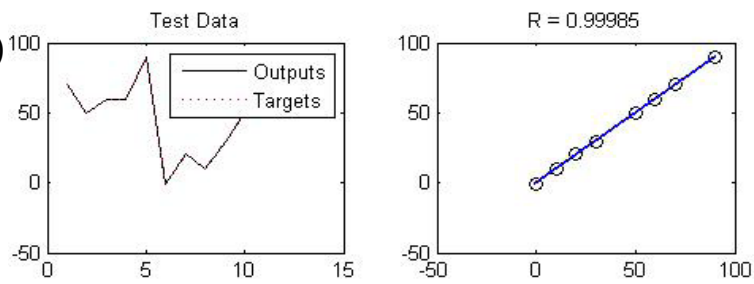

MSE $=0.29168$, RMSE $=0.54007$

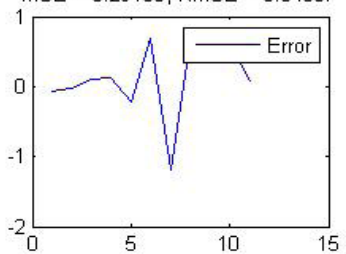

(d)
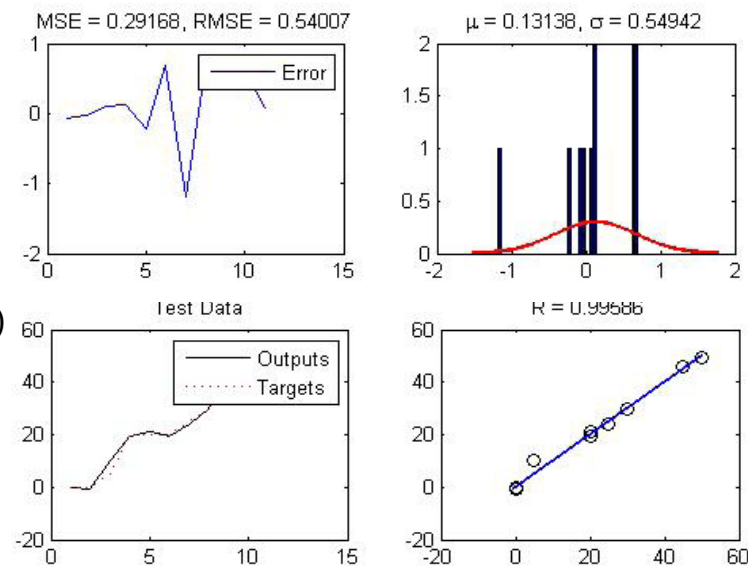

$\mathrm{K}=\mathrm{u}$. yysibb

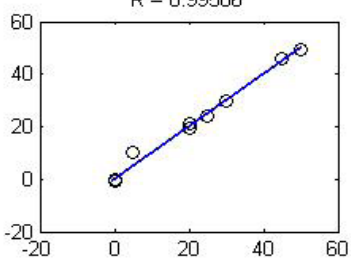

MSE $=2.7334$, RMSE $=1.6533$
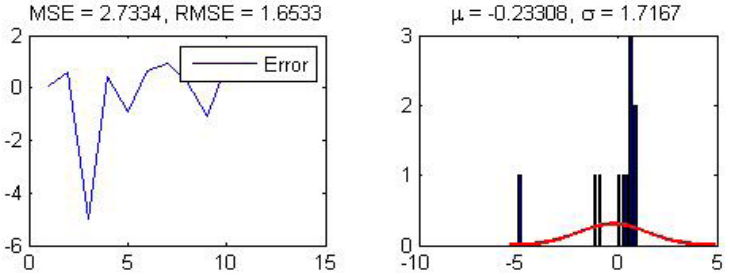

Fig. 15. Third structure/homogeneous flow regime: (a) validation data for estimation of the VF; (b) test data for estimation of the VFs; (c) validation data for estimation of WLR; (d) test data for estimation of WLR.

According to the diagrams, the RMSE for the test data is about 0.54 ; the total error would be 1.5 in the worst conditions. As was described formerly, an independent ANN is set to estimate the WLR in the system.

Based on the figures, the RMSE of the test data is 1.65 ; the error would be not $>5$ in the worst case. Table 4 demonstrates the test outputs and the target outputs.

As was described formerly, two independent ANNs were organized to estimate the VF and the WLR. There were a total of 99 data points; $10 \%$ (10 inputs) were taken for validation, $10 \%$ for the test, and 80\% (79 data) for training.

Results of the simulation for annular flow are shown in Fig. 16.

According to the diagrams, the RMSE for test data in annular flow is 0.08 . The correlation between outputs and targets is very close to one. The error approaches 2.0 for the worst case.

Results of the simulation for estimation of the WLR in annular flow are shown in Fig. 16.

According to the figures, the RMSE of the test data for estimation of the WLR in annular flow is 1.2 ; the error is $<3 \%$ in the worst scenario. The histogram of accuracy illustrates that the error is very much close to zero over a wide range of data. The results are given in Table 5 .

\section{Conclusion}

The values of WLR and VF are key parameters in multiphase flow meters. Designing and manufacturing a multiphase flow meter requires complex computation models to measure these parameters from among a set of parameters obtained from measurement sensors and gages. Nuclear multiphase flow meters are the most efficient types of flow meters utilized in petroleum industries for oil/water/gas flows. A system of multiphase flow measurement was designed by application of three thallium-doped sodium iodide scintillators and a radioactive source of ${ }^{133} \mathrm{Ba}$ installed on a plane spanning the cross section of a vertically aligned pipe carrying the three phases of oil, water, and gas. The proposed setup was simulated using the MCNP code. Transmission and scattering detectors gather data from the irradiation of the cross section of a pipe for different volumetric fractions of water, oil, and gas phases for the two most commonly occurring types of flow regimes, namely, homogeneous and annular; different structures of ANNs were developed to receive detector data as input parameters and give the flow regime, VF, and WLR as outputs. The multilayer perceptron neural network was selected to be trained by the Levenberg-Marquardt algorithm due to its efficiency.

The results showed that increasing the number of ANNs makes the system more complicated and 
(a)

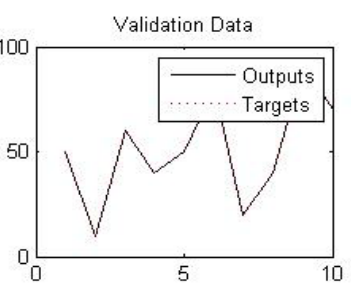

MSE $=0.0098698$, RMSE $=0.099347$

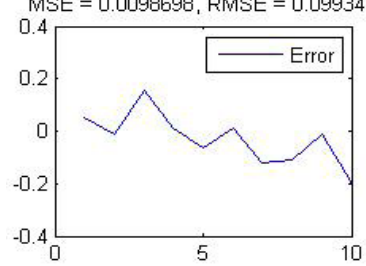

(c)

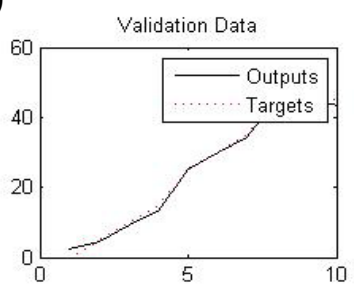

$\mathrm{MSE}=1.2811, \mathrm{RMSE}=1.1318$

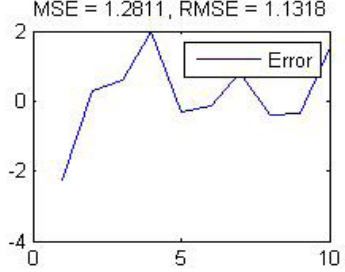

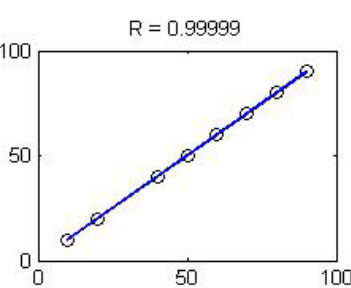
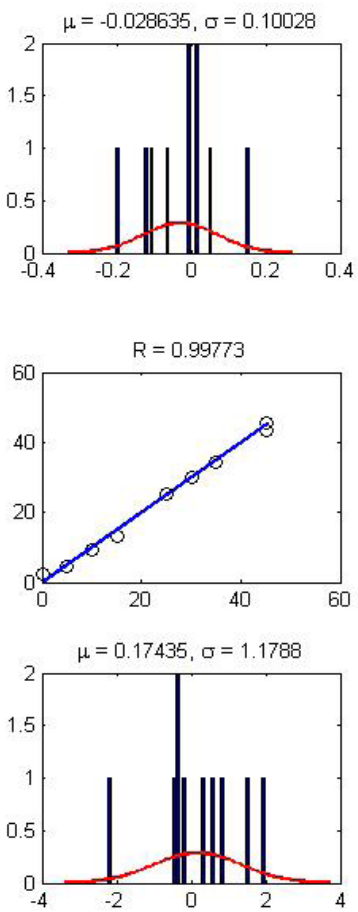

(b)
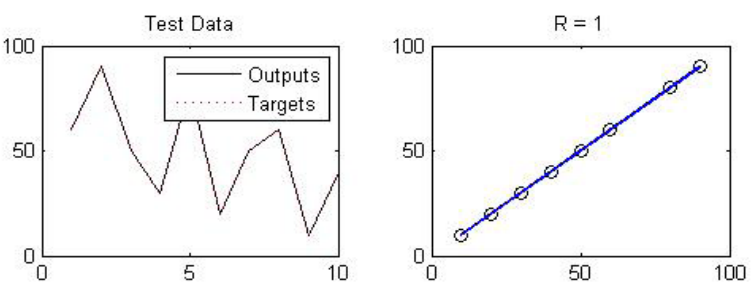

MSE $=0.006549$, RMSE $=0.080926$

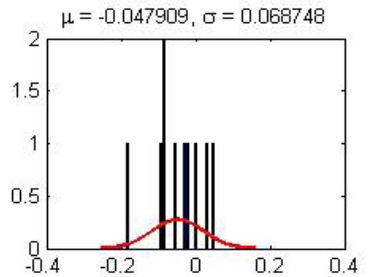

(d)
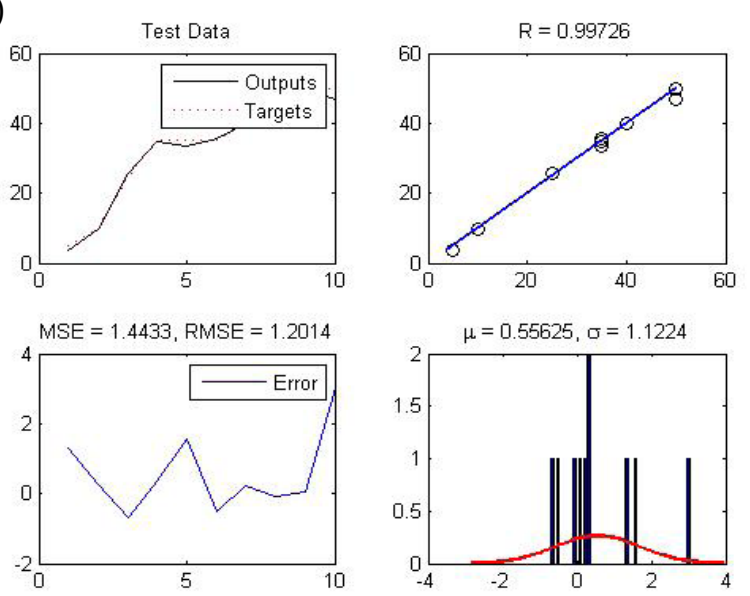

Fig. 16. Third structure/annular flow regime: (a) validation data for estimation of VFs; (b) test data for estimation of VFs; (c) validation data for estimation of WLR; (d) test data for estimation of WLR.

Table 6. Comparison of errors in the three proposed structures of ANNs

\begin{tabular}{lccccc}
\hline \multicolumn{1}{c}{ ANNs structure } & VF & \multicolumn{2}{c}{ WLR } \\
\hline First structure & 0.54 & \multicolumn{2}{c}{2.80} \\
Second structure & \multicolumn{2}{c}{0.29} & Annular & Homogeneous & Annular \\
Third structure & Homogeneous & 0.54 & 0.08 & 1.6 & 1.2 \\
\hline
\end{tabular}

decreases the available data; however, this increases the accuracy of estimation of the WLR and the GVF (see Table 6). According to the results, the maximum relative difference was observed in the scattering detector. It was clear that transmission detectors would demonstrate the difference between the flow regimes as well. It is necessary to note that the error calculated by the MCNP simulator is $<0.5 \%$ for the direct detectors (TR1 and TR2). Due to the difference between the data of the two flow regimes and the errors of data in the simulation codes of the MCNP, it was possible to separate these flow regimes. The effect of changing WLR on the efficiency for a constant VF confirms a considerable variance in the results for annular and homogeneous flows occurring in the scattering detector. There is a similar trend for the VF; hence, one can easily distinguish the changes in efficiency due to the WLR. Analysis of the simulation results revealed that in the proposed structure of the multiphase flow meter and the computation model used for simulation, the two flow regimes are simply distinguishable.

Since the available data are limited, we would have fewer data points to train each network, which, in fact, is harmful to the training process and affects the generalization capability of the system. Although the first structure provided lower accuracy, it generally was more reliable. It was trained by much more variant data. Other structures benefited from extra layers to reduce relative errors, but it led to the lack of training data, and hence, the generalization capability decreased. Each of the structures should be used depending on the application.

\section{References}

1. Scheers, A. M. (2000). An oil/water/gas composition meter based on multiple energy gamma ray absorption (MEGRA) measurement. ETDEWEB ID:20061564. 
2. Holstad, M. B. (2004). Gamma-ray scatter methods applied to industrial measurement systems. Unpublished Ph.D. thesis, Department of Physics and Technology, University of Bergen, Norway.

3. Scheers, A., \& Letton, W. (1996). An oil/water/gas composition meter based on multiple energy gamma ray absorption (MEGRA) measurement. In Proceedings of 14th North Sea Flow Measurement Workshop.

4. Blaney, S. (2008). Gamma radiation methods for clamp-on multiphase flow metering. Unpublished $\mathrm{PhD}$ thesis, Cranfield University.

5. Salgado, C., Brandao, L. E. B., Conti, C. C., \& Salgado, W. L. (2016). Density prediction for petroleum and derivatives by gamma-ray attenuation and artificial neural networks. Appl. Radiat. Isot., 116, 143-149.

6. AL-Qutami, T. A., Ibrahim, R., Ismail, I., \& Ishak, M. A. (2018). Virtual multiphase flow metering using diverse neural network ensemble and adaptive simulated annealing. Expert Syst. Appl., 93, 72-85.

7. Roshani, G., Nazemi, E., \& Roshani, M. (2017). Flow regime independent volume fraction estimation in three-phase flows using dual-energy broad beam technique and artificial neural network. Neural Comput. Appl., 28(Suppl. 1), 1265-1274.

8. Nazemi, E., Roshani, G. H., Feghhi, S. A. H., Setayeshi, S., Eftekhari Zadeh, E., \& Fatehi, A. (2016). Optimization of a method for identifying the flow regime and measuring void fraction in a broad beam gamma-ray attenuation technique. Int. J. Hydrog. Energy, 41(18), 7438-7444.

9. Johansen, G., \& Jackson, P. (2000). Salinity independent measurement of gas volume fraction in oil/gas/ water pipe flows. Appl. Radiat. Isot., 53 (4), 595-601.

10. Johansen, G. A., \& Tjugum, S. -A. (2007). Fluid composition analysis by multiple gamma-ray beam and modality measurements. In 25th International North Sea Flow Measurement Workshop, Citeseer.

11. Åbro, E., Khoryakov, V. A., Johansen, G. A., \& Kocbach, L. (1999). Determination of void fraction and flow regime using a neural network trained on simulated data based on gamma-ray densitometry. Meas. Sci. Technol., 10(7), 619-630.

12. Roshani, G. H., Feghhi, S. A. H., \& Setayeshi, S. (2015). Dual-modality and dual-energy gamma ray densitometry of petroleum products using an artificial neural network. Radiat. Meas., 82, 154-162.

13. Salgado, C. M., Brandão, L. E., Pereira, C. M., \& Salgado, W. L. (2014). Salinity independent volume fraction prediction in annular and stratified (watergas-oil) multiphase flows using artificial neural networks. Prog. Nucl. Energy, 76, 17-23.

14. Salgado, C. M., Pereira, C. M., Schirru, R., \& Brandão, L. E. (2010). Flow regime identification and volume fraction prediction in multiphase flows by means of gamma-ray attenuation and artificial neural networks. Prog. Nucl. Energy, 52(6), 555-562.

15. Srinivasan, Ravichandran, Chan, Vidhya, \& Ramakirishnan, Krishnan. (2002). Exponentiated backpropagation algorithm for multilayer feedforward neural networks. In Proceedings of the 9th International Conference on Neural Information Processing, pp. 227-331.

16. Kamarthi, S. V., \& Pittner, S. (1999). Accelerating neural network training using weight extrapolation. Neural Netw., 12(9), 1285-1299.

17. More, J. J. (1978). The Levenberg-Marquardt algorithm: Implementation and theory. In G. A. Watson (Ed.), Numerical analysis. (Lecture Notes in Mathematics, Vol. 630). Berlin, Heidelberg: Springer. 